\title{
Quimioterapia neoadyuvante en pacientes con cáncer de mama localmente avanzado, basada en el esquema ciclofosfamida, doxorrubicina, taxanos con y sin trastuzumab, de acuerdo con la sobreexpresión de HER2
}

Neoadyuvant chemotherapy in patients with breast cáncer locally advanced, treated with doxorubicin and cyclophosphamide and taxanes with or without trastuzumab

\author{
- Jesús Sánchez', Sandra Díaz², Luis Guzmán³, Diego González, Alicia Quiroga Echeverri5, David López \\ ' Internista, oncólogo clínico, magister en Epidemiología Clínica, Instituto Nacional de Cancerología (Bogotá, D.C., Colombia). \\ Cirujana, especialista en Cirugía de Seno y Tejidos Blandos, Instituto Nacional de Cancerología (Bogotá, D.C., Colombia). \\ Ginecólogo, especialista en Cirugia de Seno y Tejidos Blandos, Instituto Nacional de Cancerología (Bogota, D.C., Colombia). \\ Internista, Fellow Oncología Clinica, Instituto Nacional de Cancerologia (Bogota, D.C., Colombia). \\ (Medellin, Colombia).
}

\section{Resumen}

Introducción: a nivel mundial el empleo de esquemas de quimioterapia neoadyuvante en la enfermedad localmente avanzada de la mama es una práctica de uso rutinario que aumenta la respuesta clínica al disminuir el tamaño tumoral e incrementar la posibilidad de cirugía conservadora.

Objetivos: describir las respuestas a partir del esquema de quimioterapia neoadyuvante que se emplea de forma rutinaria AC (doxorrubicina y ciclofosfamida), más taxanos con o sin trastuzumab.

Métodos: el estudio es observacional descriptivo prospectivo, llevado a cabo entre octubre de 2012 y mayo de 2014, donde se incluyeron mujeres mayores de 18 años seleccionadas por conveniencia, con diagnóstico confirmado por histopatología de cáncer de mama epitelial infiltrante en estadios clínicos IIB, IIIA, IIIB y IIIC según la definición de la AJCC, con estado funcional menor o igual a 2 y estudios de extensión negativos para enfermedad metastásicos no mayores a dos meses.

Resultados: la muestra total fue de 109 mujeres; el promedio de edad fue de 53,3 años, la mediana de tamaño tumoral fue de $5 \mathrm{~cm}$, el $85,4 \%$ cumplieron el tratamiento propuesto, la frecuencia de los cuatro grupos intrínsecos fue en su orden luminal B1 $(41,2 \%)$, luminal A $(17,5 \%)$, triple negativo $(17,5 \%)$, luminal B2 $(16,5 \%)$ y HER2 $(7,2 \%)$. Se realizaron 31 cirugías conservadoras. Con respuesta patológica completa global del 19,7\%. La respuesta patológica fue mayor en el subgrupo triple negativo (33,3\%) y en los pacientes con sobreexpresión de HER2 (38,9\%) y muy baja en luminal A (5,6\%). Conclusiones: la quimioterapia neoadyuvante secuencial basada en AC, seguida por taxanos, asociada o no a trastuzumab según la sobreexpresión de HER2 mejora de manera importante la respuesta patológica completa y aumenta el número de cirugías conservadoras con mínima toxicidad limitante en las pacientes con cáncer de mama localmente avanzado, en el Instituto Nacional de Cancerología, comparado con la literatura internacional.

Palabras clave (DeCS): neoplasias de la mama, quimioterapia adyuvante, ciclofosfamida, doxorrubicina, taxanos, trastuzumab.

Abreviaturas: AC: doxorrubicina y ciclofosfamida, AJCC: American Joint Committee on Cancer, ECOG: Eastern Cooperative Oncology Group, NCCN: National Comprehensive Cancer Network. 


\begin{abstract}
Background: The actual use of neoadjuvant chemotherapy in breast cancer locally advanced is an indication, that raises the chance to reduce the size of the tumor and perform conservative surgery.

Objetive: Describe the response to chemotherapy AC (doxorubicin and cyclophosphamide) and taxanes with or without trastuzumab usually administered in the neoadjuvant therapy.

Methods: The study is a observational descriptive prospective, made between october 2012 and may 2014, including women older than 18 years selected by convenience, with diagnosis by histopathology of infiltrating epithelial breast cancer with clinical stage IIB, IIIA, IIIB and IIIC according to the classification of AJCC, with functional status less or equal to 2 and negative extension studies to metastatic disease no more than two months.

Results: The total sample was of 109 women, the average age was 53.3 years, the median tumor size was about $5 \mathrm{~cm}$ $85.4 \%$ of the women complete the whole treatment. The frequency of the intrinsic groups goes as it follows, luminal B1 $(41.2 \%)$, luminal A (17.5\%), triple negative (17.5\%), luminal B2 (16.5\%) and HER2 (7.2\%). 31 conservative surgeries were performed. The global pathological complete response was of $19.7 \%$. The biggest pathological response was in the triple negative subgroup 33.3\% and in patient with overexpression of HER2 the response was 38.9\%; luminal A response was quite low $5.6 \%$.

Conclusion: The neoadjuvant sequential chemotherapy based on AC protocol follow up by taxanes associated or not to trastuzumab according to the over-expression of HER2, improves pathologic complete response, and rise the number of conservative surgeries with minimal toxicity in the patients with breast cancer locally advanced in the Instituto Nacional de Cancerología, with similar results compared to the international literature.
\end{abstract}

Keywords (MeSH): Breast neoplasms, chemotherapy, adjuvant, cyclophosphamide, doxorubicin, taxanes, trastuzumab.

\section{Introducción}

Según Globocan 2018, a nivel mundial el cáncer de mama es la primera causa de mortalidad y de incidencia en mujeres.

Colombia ocupa el primer lugar en prevalencia con 39.330 casos y en incidencia con 13.380 casos nuevos; en mortalidad ocupa el cuarto puesto con 3.702 casos $^{1}$.

La supervivencia disminuye con relación al estadio clínico de la enfermedad, siendo más alto el riesgo de morir en pacientes considerados con estadios localmente avanzados (IIB, IIIA, IIIB y IIIC) ${ }^{2}$.

La quimioterapia neoadyuvante está indicada como tratamiento en cáncer de mama localmente avanzado para disminuir el tamaño tumoral y aumentar la posibilidad de cirugías conservadoras33; por otro lado, evalúa la quimiosensibilidad in vivo y se logran controlar posibles micrometástasis ${ }^{4}$. Se ha sugerido que alcanzar una respuesta patológica completa mejora la supervivencia libre de enfermedad y en algunos casos la supervivencia global, como en los tumores triple negativos y HER2 positivos 5 .

La mitad de las pacientes con cáncer de mama tratadas en el Instituto Nacional de Cancerología se diagnostica en estadios avanzados ${ }^{6}$. Un estudio realizado en la institución sobre quimioterapia neoadyuvante para cáncer de mama en estadios localmente avanzados con cuatro ciclos basados en antraciclinas mostró que el $10 \%$ de las pacientes lograron una respuesta patológica completa ${ }^{7}$. Basados en los anteriores resultados y en otros estudios ${ }^{8,9}$, se decidió administrar tratamiento neoadyuvante con esquemas a base de antraciclinas, taxanos y trastuzumab de acuerdo con la sobreexpresión de HER2.

El objetivo de este estudio es describir la respuesta clínica, patológica y la proporción de cirugías conservadoras en pacientes tratadas con quimioterapia neoadyuvante basada en esquema de cuatro ciclos de doxorrubicina y ciclofosfamida, seguido de cuatro ciclos de taxanos con o sin trastuzumab de acuerdo con sobreexpresión del oncogén HER2.

\section{Métodos}

El estudio es observacional descriptivo prospectivo, realizado entre octubre de 2012 y mayo de 2014, que incluyó mujeres seleccionadas por conveniencia, mayores de 18 años de edad con diagnóstico confirmado por histopatología de cáncer de mama epitelial infiltrante en estadios clínicos IIB, IIIA, IIIB y IIIC, según la definición del American Joint Committee on Cancer $(A J C C)^{10}$, con estado funcional menor o igual a 2, conforme con el Eastern Cooperative Oncology Group (ECOG) con estudios de extensión negativos para enfermedad metastásica no mayores a dos meses. Se excluyeron pacientes que recibieron tratamiento oncológico previo para cáncer de mama, en primer trimestre de embarazo, con contraindicación para recibir 
antraciclinas, con disfunción hepática y renal severa, sin métodos de planificación seguros y antecedentes de otros cánceres, excepto melanomas y carcinoma del cuello uterino in situ. Este estudio tuvo aprobación por el comité de ética institucional independiente del Instituto Nacional de Cancerología según acta 16 de 2012, y todas las pacientes involucradas aceptaron y firmaron de manera libre y voluntaria el consentimiento y la información para la participación en el estudio.

Las pacientes fueron tratadas con quimioterapia neoadyuvante basada en esquema de cuatro ciclos de doxorrubicina y ciclofosfamida, seguida de cuatro ciclos de taxanos con o sin trastuzumab de acuerdo con sobreexpresión del oncogén HER2. Las pacientes recibieron en el día uno $600 \mathrm{mg} / \mathrm{m}^{2}$ de ciclofosfamida más $60 \mathrm{mg} / \mathrm{m}^{2}$ de doxorrubicina en infusión endovenosa, que se repetía cada 21 días por cuatro ciclos; posteriormente, recibieron $80 \mathrm{mg} / \mathrm{m}^{2}$ de paclitaxel cada siete días durante 12 semanas o $100 \mathrm{mg} / \mathrm{m}^{2}$ de docetaxel en el primer día y luego cada tres semanas por cuatro ciclos. Los pacientes con sobreexpresión de HER2, evaluados con más de tres cruces por inmunohistoquímica (HercepTest ${ }^{\mathrm{TM}}$, Dako) o positivo para fluorescence in situ hybridization, FISH (Vysis TP53/CEP 17TM, Vysis Inc.), recibieron trastuzumab a una dosis inicial de $4 \mathrm{mg} / \mathrm{kg}$ en el día uno, seguido de $2 \mathrm{mg} / \mathrm{kg} / \mathrm{semana}$ por 12 semanas $\mathrm{u} 8 \mathrm{mg} / \mathrm{kg}$ en el día uno seguido de $6 \mathrm{mg} / \mathrm{kg}$ cada 3 semanas por 4 ciclos. Las pacientes sin expresión del HER2 solo recibieron el esquema mencionado de quimioterapia. Al finalizar el tratamiento las pacientes fueron llevadas a tratamiento quirúrgico locorregional. El tratamiento complementario se realizó siguiendo protocolos institucionales ${ }^{11}$ y los lineamientos de las guías de la National Comprehensive Cancer Network (NCCN) ${ }^{12}$.

El tiempo de seguimiento de las pacientes fue desde el momento del reclutamiento hasta la cirugía definitiva de mama. Se efectuó una evaluación inicial en donde se recolectó la edad, con estado funcional según ECOG, aspectos clínicos como clasificación TNM y estadio de la enfermedad de acuerdo con la AJCC ${ }^{10}$, características histopatológicas del tumor que incluían el tipo histológico, el grado de diferenciación, invasión linfovascular, presencia de receptores para estrógenos, progesterona y HER2 o FISH. Se categorizaron las características inmunohistoquímicas en los cuatro grupos intrínsecos, según la clasificación reportada por
Perou y Sørlie $e^{13-15}$ y se denominó luminal A: receptor de estrógenos (RE) y/o receptor de progestágenos (RP) positivo, HER2 negativo, Ki67 bajo (menor o igual del 14\%); luminal B1 (HER2 negativo): RE y/o RP positivo, HER2 negativo, Ki67 alto; luminal B2 (HER2 positivo): RE y/o RP positivo, cualquier Ki67 y sobreexpresión o amplificación de HER2; triple negativo: RE y RP negativo, HER2 negativo. En el caso del marcador para proliferación celular Ki67, fue categorizado en dos grupos: bajo para valores inferiores o iguales al 14\% y alto para niveles mayores del $14 \%{ }^{15}$. Cada control fue realizado posterior a la aplicación del esquema de quimioterapia (aproximadamente tres semanas). En cada uno se evaluaron los efectos secundarios y la respuesta clínica al tratamiento. Los efectos secundarios fueron graduados utilizando los criterios del Common Terminology Criteria for Adverse Events del Instituto Nacional de Cáncer (EE.UU.) ${ }^{16}$. La respuesta clínica fue evaluada según los parámetros de la Organización Mundial de la Salud ${ }^{17}$. Se consideró respuesta completa cuando hubo desaparición del tumor determinado por dos seguimientos clínicos separados por cuatro semanas; respuesta parcial, cuando hubo disminución del $50 \%$ o más del producto entre el diámetro mayor y el diámetro perpendicular del tumor; enfermedad estable, cuando dicho producto es menor del $50 \%$ o no hay aumento de más del 25\%; y progresión, cuando hubo un incremento del tamaño del tumor en un $25 \%$. La información fue recolectada por un especialista en oncología clínica y cirugía de tejidos blandos, con el apoyo de un coordinador operativo que evaluaba la calidad de los datos obtenidos. Los datos fueron digitados en una hoja de cálculo de Microsoft Excel ${ }^{\mathrm{TM}}$ estandarizada para su uso.

De acuerdo con la respuesta clínica del paciente, el grupo de cirugía de seno y tejidos blandos definía realizar la cirugía conservadora o radical. Con el reporte del análisis histopatológico definitivo del espécimen, el grupo investigador determinó la respuesta patológica completa, que se definió como ausencia de cáncer invasivo en la mama, pero se aceptó la presencia de cáncer in situ y en la axila ausencia de tumor ${ }^{18,19}$.

El análisis descriptivo se llevó a cabo utilizando medidas de frecuencia absoluta y relativa en el caso de las variables cualitativas. Para las variables cuantitativas se resumió la información con medidas de tendencia central (promedios o medianas) y medidas de dispersión 


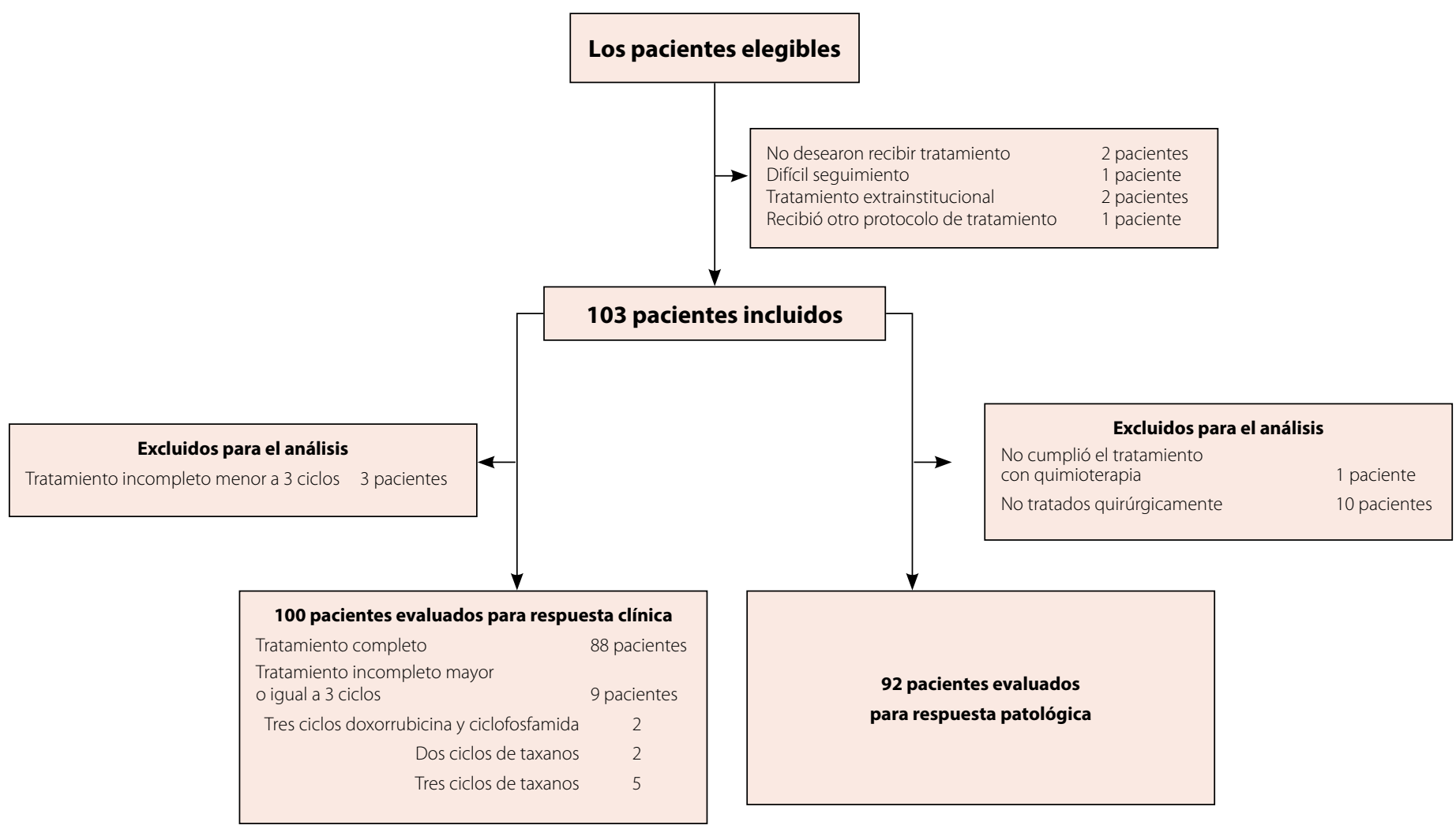

Figura 1. Selección de pacientes.

(desviaciones estándar o rangos intercuartílicos). Se validó el supuesto de normalidad mediante la prueba de Shapiro-Wilk de las variables cuantitativas. Se consideró tratamiento completo cuando se cumplió la totalidad del esquema de quimioterapia propuesto. La respuesta clínica fue evaluada en pacientes que recibieron por lo menos tres ciclos de quimioterapia, y la respuesta patológica se evalúo en pacientes que cumplieron el tratamiento y fueron llevados a cirugía. El análisis estadístico de los datos fue realizado en el software Stata Statistical Software: Release 11 (StataCorp. 2009. College Station, TX: StataCorp LP) licenciado para el Instituto Nacional de Cancerología.

\section{Resultados}

De 109 pacientes elegibles, 6 de ellas manifestaron no recibir el tratamiento o fueron tratadas en otra institución, las cuales fueron excluidas del análisis de la información (figura 1). El promedio de edad de las pacientes incluidas fue 53,3 años (desviación estándar de 9,3 años) (prueba de Shapiro-Wilk $p=0,39$ ). En la tabla 1 se resumen las características clínicas e histopatológicas de las pacientes incluidas en el estudio.
La prueba de FISH fue realizada en 11 pacientes con HER2 equívoco y en 2 pacientes con HER2 equívoco extrainstitucional, pero que en la revisión posterior fueron negativos. La mediana del tamaño tumoral fue 5,0 cm (rango intercuartílico RIQ 1,3 cm) (prueba de Shapiro-Wilk $p=0,07$ ).

La frecuencia de los cuatro grupos intrínsecos según sus características inmunohistoquímicas fue, en su orden, luminal B1 (41,2\%), luminal A (17,5\%), triple negativo (17,5\%), luminal B2 (16,5\%) y HER2 $(7,2$ $\%)$. Esta información solo fue posible en 93 pacientes debido a que no hubo disponibilidad del marcador Ki67 en 10 pacientes.

El $85,4 \%$ (88) de los pacientes cumplieron el esquema de tratamiento propuesto (figura 1), de los cuales el taxano más utilizado fue paclitaxel en el $81,1 \%$ y 18,9\% recibió docetaxel. Solo 27 pacientes recibieron tratamiento con trastuzumab.

En la tabla 2 se presenta la respuesta clínica evaluada en 100 pacientes, quienes cumplieron parcial o totalmente el tratamiento propuesto (ver métodos), según la expresión o no de HER2. Se debe destacar que tres pacientes solo completaron dos ciclos de tratamiento con 
Tabla 1. Características clínicas e histopatológicas de mujeres con cáncer de mama, pacientes tratadas con quimioterapia neoadyuvante basada en esquema de cuatro ciclos de doxorrubicina y ciclofosfamida, seguida de cuatro ciclos de taxanos con o sin trastuzumab de acuerdo con sobreexpresión del oncogén HER2 ( $n=103)$.

\begin{tabular}{|l|c|c|}
\hline \multicolumn{1}{|c|}{ Características clínicas } & n & $\%$ \\
\hline Régimen de seguridad social & 61 & 59,2 \\
\hline Contributivo & 36 & 34,9 \\
\hline Subsidiado & 6 & 5,8 \\
\hline Vinculado & & \\
\hline ECOG & 41 & 39,8 \\
\hline 0 & 62 & 60,2 \\
\hline 1 & \multicolumn{2}{|c|}{} \\
\hline Tamaño tumoral & 24 & 23,3 \\
\hline T2 & 19 & 18,4 \\
\hline T3 & 1 & 1,0 \\
\hline T4a & 59 & 57,3 \\
\hline T4b & \multicolumn{2}{|c|}{}
\end{tabular}

Estado ganglionar clinico

\begin{tabular}{|l|c|c|}
\hline No & 6 & 5,8 \\
\hline N1 & 58 & 56,4 \\
\hline N2 & 34 & 33,0 \\
\hline N3 & 5 & 4,8 \\
\hline
\end{tabular}

Estadio clínico

\begin{tabular}{|l|c|c|}
\hline IIB & 20 & 19,4 \\
\hline IIIA & 20 & 19,4 \\
\hline IIIB & 58 & 56,3 \\
\hline IIIC & 5 & 4,8 \\
\hline
\end{tabular}

\begin{tabular}{|l|c|c|}
\hline Tipo histológico & \multicolumn{2}{|l|}{} \\
\hline Carcinoma ductal & 100 & 97,1 \\
\hline
\end{tabular}

\begin{tabular}{|l|c|c|}
\hline Carcinoma ductal & 100 & 97,1 \\
\hline Carcinoma lobulillar & 2 & 1,9 \\
\hline Carcinoma mucinoso & 1 & 1,0 \\
\hline Grado de diferenciación & & \\
\hline Grado I & 4 & 3,9 \\
\hline Grado II & 68 & 66,0 \\
\hline Grado III & 31 & 30,1 \\
\hline
\end{tabular}

Receptor de estrógenos

\begin{tabular}{|l|c|c|}
\hline Positivo & 75 & 72,8 \\
\hline Negativo & 28 & 27,2 \\
\hline Receptor de progesterona & & \\
\hline Positivo & 75 & 72,8 \\
\hline Negativo & 28 & 27,2 \\
\hline Triple negativo & 17 & 16,5 \\
\hline
\end{tabular}

\begin{tabular}{|l|c|c|}
\hline \multicolumn{2}{|l|}{ Receptor HER2 } \\
\hline $0 y+$ & 72 & 69,9 \\
\hline
\end{tabular}

\begin{tabular}{|l|c|c|}
\hline $0 y+$ & 72 & 69,9 \\
\hline++ & 11 & 10,7 \\
\hline+++ & 20 & 19,4 \\
\hline FISH positivo de pacientes con receptor HER2 $++^{*}$ & 3 & 23,1 \\
\hline Ki67** & 82 & 88,2 \\
\hline Alto & 11 & 11,8 \\
\hline Bajo & \multicolumn{3}{|l|}{} \\
\hline
\end{tabular}

ECOG: Eastern Cooperative Oncology Group

FISH: fluorescence in situ hybridization

*Ver resultados.

** Los valores de Ki67 solo fueron reportados en 93 pacientes.
Tabla 2. Respuesta clínica en mujeres con cáncer de mama tratadas con quimioterapia neoadyuvante según expresión HER2

\begin{tabular}{|c|c|c|c|c|c|c|}
\hline \multirow[t]{2}{*}{$\begin{array}{l}\text { Respuesta } \\
\text { clínica }\end{array}$} & \multicolumn{2}{|c|}{$\begin{array}{c}\text { Esquema de } \\
\text { tratamiento } \\
\text { completo' } \\
(n=88) \\
\%(n)\end{array}$} & \multicolumn{2}{|c|}{$\begin{array}{c}\text { Esquema de } \\
\text { tratamiento } \\
\text { incompleto } \\
(n=12) \\
\%(n)\end{array}$} & \multicolumn{2}{|c|}{$\begin{array}{l}\text { Esquema de } \\
\text { tratamiento } \\
(n=100) \\
\%(n)\end{array}$} \\
\hline & HER2- & HER2+ & HER2- & HER2+ & HER2- & HER2+ \\
\hline Completa & $21,2(14)$ & $22,7(5)$ & $9,1(1)$ & $100,0(1)$ & $19,5(15)$ & 26,1 (6) \\
\hline Parcial & $53,0(35)$ & $50,0(11)$ & $36,4(4)$ & - & $50,7(39)$ & $47,8(11)$ \\
\hline Estable & $24,2(16)$ & $24,3(6)$ & $27,3(3)$ & - & $24,7(19)$ & $26,1(6)$ \\
\hline Progresión & $1,5(1)$ & - & $27,3(3)$ & - & $5,2(4)$ & - \\
\hline Total & $100(66)$ & $100(22)$ & $100(11)$ & $100(1)$ & $100(77)$ & $100(23)$ \\
\hline
\end{tabular}

Esquema de cuatro ciclos de doxorrubicina y ciclofosfamida, seguido de cuatro ciclos de

taxanos con o sin trastuzumab de acuerdo con sobreexpresión del oncogén HER2.

${ }^{2}$ Esquema parcial de tratamiento: dos pacientes tres ciclos doxorrubicina y ciclofosfamida, tres pacientes cuatro ciclos de doxorrubicina y ciclofosfamida, dos pacientes dos ciclos de taxanos y cinco pacientes recibieron tres ciclos de taxanos.

Sumatoria de ambos esquemas de tratamiento de los numerales 1 y 2

doxorrubicina y ciclofosfamida, que se consideró insuficiente para determinar respuesta. Se observó una mayor respuesta clínica completa en pacientes con expresión del grupo intrínseco luminal B1 (45,0\%). La progresión de la enfermedad fue más frecuente en pacientes con grupo intrínseco triple negativo $(75,0 \%)$ (tabla 3$)$.

Del total de pacientes, 92 (89,3\%) fueron operadas en la institución. De estas, 60 pacientes fueron tratadas con cirugía radical modificada y una paciente con cirugía radical de Halsted. Previo a la cirugía, 27 pacientes habían sido consideradas inoperables y otras 34 para cirugía radical.

En el caso de la cirugía conservadora, esta fue realizada en 31 pacientes, de las cuales 29 fueron manejadas con cuadrantectomía más vaciamiento ganglionar axilar, y 2 pacientes fueron tratadas con mamoplastia oncológica más vaciamiento ganglionar axilar. Sin embargo, antes de la quimioterapia neoadyuvante, 11 pacientes habían sido consideradas inoperables y 20 habían sido elegibles para cirugía radical.

La respuesta patológica completa global fue del 19,7\% evaluada en 91 pacientes. Una paciente fue excluida del análisis de la respuesta patológica, a pesar de haber sido operada, debido a que solo recibió dos ciclos de doxorrubicina y ciclofosfamida. La tabla 4 presenta la respuesta patológica observada en pacientes de acuerdo con el cumplimiento del esquema de tratamiento y la expresión de HER2.

La toxicidad hematológica grado 3 y 4 fue baja (tabla 6); neutropenia, 3 y 1 pacientes; y trombocitopenia, 1 paciente. En cuanto a la toxicidad no hematológica, astenia, náuseas, vómito y diarrea fueron las más frecuentes. 
Tabla 3. Respuesta clínica en mujeres con cáncer de mama, pacientes tratadas con quimioterapia neoadyuvante según expresión de HER2, discriminación de acuerdo con grupos intrínsecos $(n=94)$

\begin{tabular}{|l|c|c|c|c|}
\hline & Completa & Parcial & Estable & Progresión \\
\hline Luminal A \%(n) & $15,0(3)$ & $17,4(8)$ & $20,8(5)$ & - \\
\hline Luminal B1*\%(n) & $45,0(9)$ & $43,5(20)$ & $41,7(10)$ & $25,0(1)$ \\
\hline Luminal B2*\%(n) & $30,0(6)$ & $13,0(6)$ & $16,7(4)$ & - \\
\hline HER2 \%(n) & - & $10,9(5)$ & $8,3(2)$ & 16 \\
\hline Triple negativo \%(n) & $10,0(2)$ & $15,2(7)$ & $12,5(3)$ & $75,0(3)$ \\
\hline Total & 20 & 46 & 24 & 15 \\
\hline
\end{tabular}

Nota: La respuesta clínica según el grupo intrínseco fue calculada en 94 pacientes debido a que 6 pacientes no tenían disponibilidad de los niveles de Ki67.

*Se define luminal B1 con HER2 negativo y un Ki67 mayor o igual al 14 \% y luminal B2 con sobreexpresión de HER2 y cualquier valor de Ki67. Para ambos grupos intrínsecos, debe existir positividad de al menos uno de los receptores de estrógeno o de progesterona.

Tabla 4. Respuesta patológica en mujeres con cáncer de mama, pacientes tratadas con quimioterapia neoadyuvante según expresión HER2

\begin{tabular}{|c|c|c|c|c|c|c|}
\hline \multirow[t]{2}{*}{ Respuesta patológica } & \multicolumn{2}{|c|}{$\begin{array}{c}\text { Esquema de tratamiento } \\
\text { completo }(\mathbf{n}=85) \\
\%(n)\end{array}$} & \multicolumn{2}{|c|}{$\begin{array}{l}\text { Esquema de tratamiento } \\
\text { incompleto }{ }^{2}(n=6) \\
\%(n)\end{array}$} & \multicolumn{2}{|c|}{$\begin{array}{c}\text { Esquema de tratamiento }{ }^{3} \\
\qquad \begin{array}{c}(\mathbf{n}=91) \\
\%(n)\end{array}\end{array}$} \\
\hline & HER2- & HER2+ & HER2- & HER2+ & HER2- & HER2+ \\
\hline Sí & $15,6(10)$ & $33,3(7)$ & $16,6(1)$ & - & $15,7(11)$ & $33,3(7)$ \\
\hline No & $84,4(54)$ & $66,7(14)$ & $83,3(5)$ & - & $84,3(59)$ & $66,7(14)$ \\
\hline Total & $100(64)$ & $100(21)$ & $100(6)$ & - & $100(70)$ & $100(21)$ \\
\hline
\end{tabular}

Un paciente se excluyó porque recibió solo dos ciclos de quimioterapia.

'Esquema de cuatro ciclos de doxorrubicina y ciclofosfamida, seguido de cuatro ciclos de taxanos con o sin trastuzumab de acuerdo con sobreexpresión del oncogén HER2.

${ }^{2}$ Esquema parcial de tratamiento: un paciente recibió tres ciclos de taxanos; un paciente, cuatro ciclos de doxorrubicina y ciclofosfamida; dos pacientes, tres ciclos de doxorrubicina y ciclofosfamida; y dos pacientes, 2 ciclos de taxanos.

${ }_{3}^{3}$ Sumatoria de ambos esquemas de tratamiento de los numerales 1 y 2.

\section{Discusión}

La quimioterapia neoadyuvante permite evaluar sensibilidad a la quimioterapia y convertir lesiones inoperables en operables, por lo que es la base del tratamiento en cáncer de mama localmente avanzado ${ }^{3,20}$.

El presente reporte de 100 pacientes con cáncer de mama localmente avanzado (figura 1), con mediana de edad 53,3 años y mediana del tamaño del tumor de $5 \mathrm{~cm}$, se muestra un predominio de T4b $(57,3 \%)$, ganglio positivo en $94,2 \%$ (tabla 1), a diferencia de los trabajos en la literatura que muestran estadios tempranos, NSABP 18 informó T2: 59,0\% y ganglio positivo 26,0\%, NSABP 27: T2 58\% y ganglio positivo $30 \%$ 9,21,24, Gepartrio T2: 61,7, N1: 53\%19.

Mientras que en los estudios con cáncer de mama localmente avanzado en tratamiento neoadyuvante con trastuzumab, como en el estudio NOAH con el uso de paclitaxel-doxorrubicina, paclitaxel, ciclofosfamida, metotrexate, 5 fluorouracilo asociado a trastuzumab, reportó T4: 42\% y N2: 39\% ${ }^{22}$. En nuestro estudio T4 representó el 58,3\% y N2: 33\%.

El $88 \%$ de nuestros pacientes recibieron el esquema propuesto y la respuesta clínica completa fue del $21,0 \%$, global del $71,0 \%$, discriminada en pacientes HER2+ fue del $6 \%$ y $18 \%$, respectivamente. Un estudio realizado en el INC ${ }^{8}$ encontró una PRC del 8,2\% después de quimioterapia neoadyuvante basada en antraciclinas. En el estudio NSABP $27^{9}$ la respuesta clínica global fue del 90,7\% y completa del 63,6\%, explicado por el predominio de estadios tempranos. El estudio GeparTrio ${ }^{19}$ incluyó algunos pacientes T4 y la respuesta clínica completa fue del 19\% con el esquema TAC, similar a la reportada por nosotros.

En el análisis por subgrupos intrínsecos la respuesta clínica global se concentró en el grupo luminal B1 (receptor hormonal positivo, HER2 negativo) 30,8\%, tabla 3 , posiblemente porque reunía la mitad de la población y otras poblaciones estaban menos representadas, mientras que la progresión clínica durante tratamiento fue más frecuente en el grupo triple negativo, que está acorde con su biología más agresiva.

En cuanto a la cirugía, la quimioterapia cambió la intención inicial y a 38 pacientes considerados inoperables fue posible llevarlos a procedimientos quirúrgicos, y de 34 mastectomías radicales se pasó a una. La cirugía conservadora fue posible en el 33,7 \% de los pacientes; en estudios con cáncer más temprano y sin tratuzumab como NSABP B-27 fue del 62\% (9), en GeparTrio del $57,3 \%$ y mastectomías del 39,6\% (19); en estudios con trastuzumab como CHER-LOB el porcentaje de conversión de mastectomía a cirugía conservadora fuedel 61,9\% (8), GeparQuattro la cirugía conservadora fue del $63,1 \%$ de los pacientes HER2 positivos y un $64,7 \%$ en HER2 negativo (23). 
Tabla 5. Respuesta patológica en mujeres con cáncer de mama, pacientes tratadas con quimioterapia neoadyuvante según grupos intrínsecos

\begin{tabular}{|l|c|c|}
\hline & Si & No \\
\hline Luminal A \%(n) & $5,6(1)$ & $20,3(14)$ \\
\hline Luminal B1*\%(n) & $22,2(4)$ & $47,8(33)$ \\
\hline Luminal B2*\%(n) & $16,7(3)$ & $15,9(11)$ \\
\hline HER2 \%(n) & $22,2(4)$ & $4,4(3)$ \\
\hline Triple negativo \%(n) & $33,3(6)$ & $11,6(8)$ \\
\hline
\end{tabular}

* Se define luminal B1 con HER2 negativo y un Ki67 mayor o igual al 14\% y luminal B2 con sobreexpresión de HER2 y cualquier valor de Ki67. Para ambos grupos intrínsecos, debe existir positividad de al menos uno de los receptores de estrógeno o de progesterona.

La respuesta patológica fue mayor en el subgrupo triple negativo $33,3 \%$ y en los pacientes con sobreexpresión de HER2 38,9\% y muy baja en luminal A $5,6 \%$ (tabla 5), similar a la reportado en la literatura, como en el estudio Neoaltto $36,5 \%$ en el grupo tratado con trastuzumab (25); en el estudio CHER-LOB en el brazo con trastuzumab la respuesta patológica fue del 25\%. En el estudio GeparQuattro²3, que incluyó cáncer localmente avanzado, la respuesta patológica global fue del $31,7 \%$ en HER2 positivo y del 15,7\% en HER2 negativo, también muy similar a lo informado en el presente trabajo.

En el estudio NSABP 27(9)411, la respuesta clínica global fue del $90,7 \%$ y completa del $63,6 \%$, la respuesta patológica completa del $26,1 \%$, cirugía conservadora en el $62 \%$, explicado por el predominio de estadios tempranos.

El estudio GeparTrio ${ }^{19}$ incluyó algunos pacientes T4 y comparó las respuestas a TAC con los respondedores a un esquema con vinorelbine-capecitabine la respuesta clínica completa fue del $19 \%$ con el esquema TAC y la respuesta patológica completa del 5,3\%. La cirugía conservadora se logró en un $57,3 \%$ y mastectomía en el 39,6\%, algunos datos comparables a los nuestros.

En el estudio CHER-LOB, en el brazo con trastuzumab la respuesta patológica fue del $25 \%$, similar a la reportada en el presente estudio, y el porcentaje de conversión de mastectomía a cirugía conservadora fue del $61,9 \%$, pero la mayoría fueron estadios IIA, IIB, y el estadio IIIA fue menos del $20 \%$, a diferencia de este trabajo donde la mayoría son estadios localmente avanzados.

En el estudio GeparQuattro ${ }^{23}$, que incluyó cáncer localmente avanzado, la respuesta patológica completa global fue del $31,7 \%$ en HER2 positivo y del $15,7 \%$ en HER2 negativo, similar a la informada en el presente estudio. La cirugía conservadora fue posible en el
Tabla 6. Toxicidad asociada al tratamiento

\begin{tabular}{|c|c|c|c|c|}
\hline & $\begin{array}{l}\text { Sin } \\
\text { toxicidad }\end{array}$ & $\begin{array}{c}\text { Grado } \\
1 \text { y } 2\end{array}$ & $\begin{array}{c}\text { Grado } \\
3\end{array}$ & $\begin{array}{c}\text { Grado } \\
4\end{array}$ \\
\hline \multicolumn{5}{|l|}{ Hematológicos } \\
\hline Anemia & 769 & 3 & - & - \\
\hline Leucopenia & 762 & 10 & - & - \\
\hline Neutropenia & 746 & 18 & 7 & 1 \\
\hline Trombocitopenia & 771 & - & 1 & - \\
\hline \multicolumn{5}{|l|}{ No hematológicos } \\
\hline Alopecia & 35 & 737 & - & - \\
\hline Astenia & 652 & 116 & 4 & - \\
\hline Colitis & 769 & 3 & - & - \\
\hline Diarrea & 695 & 71 & 6 & - \\
\hline Elevación de creatinina & 772 & - & - & - \\
\hline Elevación de fosfatasa alcalina & 771 & 1 & - & - \\
\hline Elevación de transaminasas & 751 & 21 & - & - \\
\hline Fiebre & 770 & 2 & - & - \\
\hline Mucositis & 694 & 78 & - & - \\
\hline Náuseas & 540 & 225 & 7 & - \\
\hline $\begin{array}{l}\text { Reacción en el sitio } \\
\text { de la inyección }\end{array}$ & 761 & 10 & 1 & - \\
\hline Toxicidad cardíaca & 772 & - & - & - \\
\hline Toxicidad hepática & 771 & 1 & - & - \\
\hline Vómito & 666 & 94 & 12 & - \\
\hline
\end{tabular}

$63,1 \%$ de los pacientes HER2 positivo y del $64,7 \%$ en HER2 negativo.

Respecto a la toxicidad en el estudio NSABP B-2721, la tasa de neutropenia febril fue significativamente más frecuente en el grupo que recibió $A C$ y docetaxel neoadyuvante en comparación con el AC solo ( $21 \%$ vs. $7 \%$ ), que en nuestro caso fue comparativamente mucho menor en ambos esquemas, posiblemente por el uso de factores estimulantes de colonias en pacientes mayores de 65 años y con docetaxel, esquema elegido para las pacientes que viven fuera de la ciudad. En cuanto a la toxicidad no hematológica, esta fue menor a la reportada en la literatura por el uso de setrones e inhibidores de la sustancia P.

Por el uso de cardiotóxicos (doxorrubicina y trastuzumab), en nuestro estudio se hizo búsqueda activa de disfunción ventricular sin documentar casos de deterioro en la FEVI o algún otro tipo de toxicidad cardíaca que en términos generales es alrededor del 3\% (35). Adicionalmente, el anterior estudio reportó tasas de toxicidades grado 3, como estomatitis y diarrea mayor, en el grupo que recibió antracíclicos (esquema FAC) que con el paclitaxel; sin embargo, estas son mayores a las reportadas en nuestros pacientes, posiblemente explicado por el uso de fluorinado. Por otra parte, los eventos reportados grado 3/4 por náuseas y vómito fueron similares. 
El presente estudio muestra que el esquema de administración de AC cada tres semanas seguido por paclitaxel es eficaz y seguro, apoyado en la buena tasa de respuesta patológica y en la baja incidencia de eventos adversos serios, especialmente de origen hematológico.

Los estudios observacionales de este tipo tienen limitaciones importantes, como la falta de grupo comparador, lo que impide impactar de forma contundente en la práctica clínica, aunque en el presente estudio permite sugerir algunas conclusiones. Además, también se presentó una pérdida de seguimiento mayor a la esperada, explicada en parte por problemas de contratación con las administradoras de salud.

\section{Conclusión}

La quimioterapia neoadyuvante secuencial basada en antraciclinas, seguida por taxanos asociada o no a trastuzumab según la sobreexpresión de HER2 mejora de manera importante la respuesta clínica-patológica y aumenta el número de cirugías conservadoras con mínima toxicidad limitante en las pacientes con cáncer de mama localmente avanzado en el Instituto Nacional de Cancerología.

Financiación: este trabajo ha sido desarrollado con apoyo financiero del Instituto Nacional de Cancerología ESE.

Conflictos e independencia editorial: los autores de esta investigación manifiestan que no tienen algún tipo de conflicto de interés relacionado con el objetivo del trabajo.

\section{Referencias}

1. Bray F, Ferlay J, Soerjomataram I, et at. Global cancer statistics 2018: Globocan estimates of incidence and mortality worldwide for 36 cancers in 185 countries. CA Cancer J Clin. 2018; 68:394-424.

2. Edge SB, Compton CC. The American Joint Committee on Cancer: the 7th edition of the AJCC cancer staging manual and the future of TNM. Annals of Surgical Oncology. 2010:1471-4.

3. Boughey JC, Peintinger F, Meric-Bernstam F, Perry AC, Hunt KK, Babiera G V, et al. Impact of preoperative versus postoperative chemotherapy on the extent and number of surgical procedures in patients treated in randomized clinical trials for breast cancer. Ann Surg. 2006;244(3):464-70.

4. Cancerología IN de. Primary systemic therapy of breast cancer. The Oncologist. Bogotá, United States; 2011. 31-84 p.

5. Cortazar $P$, Zhang $L$, Untch $M$, Mehta K, Costantino JP, Wolmark N, et al. Pathological complete response and long-term clinical benefit in breast cancer: The CTNeoBC pooled analysis. Lancet. 2014;384(9938):164-72.

6. Instituto Nacional de Cancerología. Anuario estadístico 2012. Bogotá; 2012. 110 p.

7. Sánchez J. Experiencia clínica del uso de quimioterapia neoadyuvante con esquema $A C$ en pacientes con cáncer de mama. Rev Colomb Cancerol. 2014;18(4):211-2.

8. Buzdar AU, Ibrahim NK, Francis D, Booser DJ, Thomas ES, Theriault $\mathrm{RL}$, et al. Significantly higher pathologic complete remission rate after neoadjuvant therapy with trastuzumab, paclitaxel, and epirubicin chemotherapy: results of a randomized trial in human epidermal growth factor receptor 2-positive operable breast cancer. J Clin Oncol. 2005;23(16):3676-85.

9. Bear HD, Anderson S, Smith RE, Geyer CEJ, Mamounas EP, Fisher B, et al. Sequential preoperative or postoperative docetaxel added to preoperative doxorubicin plus cyclophosphamide for operable breast cancer: National Surgical Adjuvant Breast and Bowel Project Protocol B-27. J Clin Oncol. 2006;24(13):2019-27.

10. Stephen Edge. AJCC Cancer Staging Manual. 7th ed. Edge $S$, Byrd DR, Compton CC, Fritz AG, Greene FL, Trotti A, editors. Springer-Verlag New York; 2010. 648 p.
11. Ministerio de Salud y Protección Social, Colciencias, Instituto Nacional de Cancerología, ESE-Fedesalud. Guía de atención integral para la detección temprana, tratamiento integral, seguimiento y rehabilitación del cáncer de mama. Bogotá; 2013. 929 p.

12. Gradishar WJ, Anderson BO, Blair SL, Burstein HJ, Cyr A, Elias $A D$, et al. Breast cancer version 3.2014. J Natl Compr Canc Netw. 2014;12(4):542-90.

13. Sorlie T, Perou CM, Tibshirani R, Aas T, Geisler $S$, Johnsen $H$, et al. Gene expression patterns of breast carcinomas distinguish tumor subclasses with clinical implications. Proc Natl Acad Sci USA [Internet]. 2001;98(19):10869-74. Disponible en: http:// www.pubmedcentral. nih.gov/articlerender.fcgi?artid=58566\& tool=pmcentrez\&rendertype $=$ abstract

14. Perou CM, Sørlie T, Eisen MB, van de Rijn M, Jeffrey SS, Rees $C$ $a$, et al. Molecular portraits of human breast tumours. Nature. 2000;406(6797):747-52.

15. Goldhirsch A, Wood WC, Coates AS, Gelber RD, Thurlimann $\mathrm{B}$, Senn $\mathrm{H}-\mathrm{J}$. Strategies for subtypes--dealing with the diversity of breast cancer: highlights of the St. Gallen International Expert Consensus on the Primary Therapy of Early Breast Cancer 2011. Annals of Oncology: official journal of the European Society for Medical Oncology. 2011:1736-47.

16. Cancer Therapy Evaluation Program. Common Terminology Criteria for Adverse Events, Version 3.0 [Internet]. 3rd ed. 2006. 76 p. Disponible en: http://ctep.cancer.gov/protocolDevelopment/electronic_applications/docs/ctcaev3.pdf

17. Hunter RD. WHO handbook for reporting results of cancer treatment [internet]. International Journal of Radiation Biology. 1980. p. 45 p. Disponible en: http://informahealthcare.com/ doi/abs/10.1080/09553008014551861

18. Cortazar P, Geyer CEJ. Pathological complete response in neoadjuvant treatment of breast cancer. Ann Surg Oncol. 2015; 22(5):1441-6.

19. von Minckwitz $G$, Kummel $S$, Vogel $P$, Hanusch $C$, Eidtmann $\mathrm{H}$, Hilfrich J, et al. Neoadjuvant vinorelbine-capecitabine versus docetaxel-doxorubicin-cyclophosphamide in early nonresponsive breast cancer: phase III randomized GeparTrio trial. J Natl 
Cancer Inst. 2008;100(8):542-51.

20. Schwartz GF, Hortobagyi GN. Proceedings of the consensus conference on neoadjuvant chemotherapy in carcinoma of the breast, april 26-28, 2003, Philadelphia, Pennsylvania. Cancer. 2004;100(12):2512-32

21. Wolmark N, Wang J, Mamounas E, Bryant J, Fisher B. Preoperative chemotherapy in patients with operable breast cancer: nine-year results from National Surgical Adjuvant Breast and Bowel Project B-18. J Natl Cancer Inst Monogr. 2001;(30):96102.

22. Gianni L, Eiermann W, Semiglazov V, Lluch A, Tjulandin S, Zambetti $M$, et al. Neoadjuvant and adjuvant trastuzumab in patients with HER2-positive locally advanced breast cancer (NOAH): follow-up of a randomised controlled superiority trial with a parallel HER2-negative cohort. Lancet Oncol. England; 2014;15(6):640-7.

23. Untch M, Rezai M, Loibl S, Fasching PA, Huober J, Tesch $H$, et al. Neoadjuvant treatment with trastuzumab in HER2-positive breast cancer: results from the GeparQuattro study. J Clin Oncol. 2010;28(12):2024-31.

24. Rastogi $P$, Anderson SJ, Bear HD, Geyer CE, Kahlenberg MS, Robidoux $A$, et al. Preoperative chemotherapy: updates of National Surgical Adjuvant Breast and Bowel Project Protocols B-18 and B-27. J Clin Oncol. 2008;26(5):778-85.

25. Baselga J, Bradbury I, Eidtmann H, Di Cosimo S, de Azambuja E, Aura C, et al. Lapatinib with trastuzumab for HER2-positive early breast cancer (NeoALTTO): a randomised, open-label, multicentre, phase 3 trial. Lancet. 2012;379(9816):633-40 